\title{
The Overdense Environments of WISE-Selected, Ultra-Luminous, High-Redshift AGN in the Submillimeter
}

OPEN ACCESS

Edited by:

Paola Marziani,

Osservatorio Astronomico di Padova

(INAF), Italy

Reviewed by:

Mauro D'Onofrio,

Università degli Studi di Padova, Italy

Daniela Bettoni,

Osservatorio Astronomico di Padova

(INAF), Italy

Giulia Rodighiero,

Dipartimento di Fisica e Astronomia,

Università degli Studi di Padova, Italy

${ }^{*}$ Correspondence:

Suzy F. Jones

suzy.jones@chalmers.se

Specialty section:

This article was submitted to

Milky Way and Galaxies,

a section of the journal

Frontiers in Astronomy and Space

Sciences

Received: 12 July 2017 Accepted: 07 November 2017 Published: 21 November 2017

Citation:

Jones SF (2017) The Overdense Environments of WISE-Selected,

Ultra-Luminous, High-Redshift AGN in the Submillimeter.

Front. Astron. Space Sci. 4:51. doi: 10.3389/fspas.2017.00051

\begin{abstract}
Suzy F. Jones *
Department of Space, Earth, and Environment, Chalmers University of Technology, Onsala Space Observatory, Onsala, Sweden
\end{abstract}

The environments around WISE-selected hot dust obscured galaxies (Hot DOGs) and WISE/radio-selected active galactic nuclei (AGNs) at average redshifts of $z=2.7$ and $z=1.7$, respectively, were found to have overdensities of companion Submillimeter-selected sources. The overdensities were of $\sim 2-3$ and $\sim 5-6$, respectively, compared with blank field submm surveys. The space densities in both samples were found to be overdense compared to normal star-forming galaxies and Submillimeter galaxies (SMGs). All of the companion sources have consistent mid-IR colors and mid-IR to submm ratios to SMGs. Monte Carlo simulations show no angular correlation, which could indicate protoclusters on scales larger than the SCUBA-2 1.5 arcmin scale maps. WISE-selected AGNs appear to be good indicators of overdense areas of active galaxies at high redshift.

Keywords: galaxies: active, galaxies: clusters: general, galaxies: high-redshift, galaxies: quasars: general, infrared: galaxies, submillimeter: galaxies

\section{INTRODUCTION}

There has been previous evidence of overdense regions around high-redshift luminous galaxies (Blain et al., 2004; Borys et al., 2004; Farrah et al., 2006; Scott et al., 2006; Gilli et al., 2007; Magliocchetti and Brüggen, 2007; Chapman et al., 2009; Hickox et al., 2009; Cooray et al., 2010; Hickox et al., 2012; Donoso et al., 2014; Umehata et al., 2014). The evolution and properties of active galactic nuclei (AGNs) are connected to their host galaxies properties and their environments. The environments around high-redshift radio galaxies (HzRGs) and radio loud AGNs (RLAGNs) ${ }^{1}$ have also been found to be overdense in dusty companions (Stevens et al., 2003; De Breuck et al., 2004; Falder et al., 2010; Galametz et al., 2010, 2012; Stevens et al., 2010; Mayo et al., 2012; Wylezalek et al., 2013; Dannerbauer et al., 2014; Hatch et al., 2014; Rigby et al., 2014; Wylezalek et al., 2014). RLAGNs are mostly found in giant, massive, elliptical galaxies and are in very dense environments (Matthews et al., 1964; Best et al., 2005; Donoso et al., 2010; Wylezalek et al., 2013). These could be signposts of high-redshift galaxy clusters (Wylezalek et al., 2013; Hatch et al., 2014).

Overdense environments around AGNs could be evidence for massive dark matter halos and highlight the bias of this distribution as compared with the underlying dark matter distribution. It is important to understand the evolution of the underlying dark matter distribution because the formation of dark matter halos are connected to the formation of galaxies and therefore to the

${ }^{1}$ RLAGNs can be classified by $S_{5 \mathrm{GHz}} / \mathrm{S}_{B} \geq 10$ (Kellermann et al., 1989; Miller and Goodrich, 1990; Urry and Padovani, 1995) and $\mathrm{L}_{500 \mathrm{MHz}} \geq 10^{27.5} \mathrm{~W} \mathrm{~Hz}^{-1}$ (Donoso et al., 2010; Hatch et al., 2014). 
properties of galaxies in the local Universe (Mo and White, 2002; Wechsler et al., 2006; Bett et al., 2007; Gao et al., 2007; Jing et al., 2007; Wetzel et al., 2007; Fakhouri and Ma, 2009; Fakhouri et al., 2010; Faltenbacher and White, 2010; Wake et al., 2012; Avila et al., 2014). Studying galaxies at higher redshifts can reveal the processes that have formed galaxies around us today.

The question of why AGN lie in dense regions and how they are affected by their environments is still debated. One suggestions is that there is hot halo mode accretion (cooling of the hot viralised atmospheres) in dense environments and cold mode accretion (galaxies accrete gas directly from cold dense intergalactic filaments) in less dense environments (Coil et al., 2009; Fanidakis et al., 2011). Coldwell and Lambas (2006) concluded that the number density of galaxies around AGN is similar to that around normal galaxies. Likewise Miller et al. (2003) found no difference in the local density around field galaxies and AGN. This is in contrast to results from for example, Kauffmann et al. (2004), Ruderman and Ebeling (2005), Serber et al. (2006), and Georgakakis et al. (2007) that indicate higher galaxy density around AGN. Quasars $\left(\mathrm{M}_{\mathrm{i}} \leq-22, z \leq 0.4\right)$ have been found to have high density regions around them at radii between $25 \mathrm{kpc}$ and $1 \mathrm{Mpc}$, with the overdensity being greatest closest to the quasar (Serber et al., 2006). Hatch et al. (2011) also found overdense regions surround $\mathrm{H} \alpha$ emitters at $z \sim 2$ that could be signposts to protocluster environments. They concluded that galaxy growth was accelerated in dense environments in the early Universe. Simulations have shown small-scale excess at scales below $\sim 100 \mathrm{~h}^{-1} \mathrm{kpc}$ (Degraf et al., 2011), consistent with observational evidence (Hennawi et al., 2006; Myers et al., 2007).

The clustering of galaxies is important because it signposts the environment richness of the galaxies. Galaxies reside in dark matter halos and the mass of the dark matter halos determines the clustering strength and the strength of the biasing (Strauss and Willick, 1995). Clustering can be used to measure dark matter halo mass and how the galaxies populate the dark matter halos (Coil, 2013), and constrain cosmological parameters in galaxy evolution models for example baryon density (Davis et al., 1985; Kauffmann et al., 1993; Navarro et al., 1996; Springel et al., 2005; Coil, 2013).

Studying the environments of Hot DOGs and WISE/radio AGNs will help to understand the evolution of galaxies and the link with their host galaxy.

\section{SAMPLES}

Advances in infrared (IR) telescope technology like the NASA's Wide-Field Infrared Survey Explorer (WISE; Wright et al., 2010) have enabled observations of luminous AGN that have been difficult to find with previous IR missions. WISE is able to find luminous, dusty, high-redshift, active galaxies because the hot dust heated by AGN and/or starburst activity can be traced using the WISE $12 \mu \mathrm{m}$ (W3) and $22 \mu \mathrm{m}$ (W4) bands. Eisenhardt et al. (2012), Bridge et al. (2013), and Lonsdale et al. (2015) have shown that WISE can find different classes of interesting, luminous, high-redshift, dust-obscured AGN.
Submillimeter observations using the James Clerk Maxwell Telescope (JCMT) Submillimeter Common-User Bolometer Array 2 (SCUBA-2) (Holland et al., 2013) of two subsamples of Wide-Field Infrared Survey Explorer (WISE; Wright et al., 2010) selected galaxies found overdensities of Submillimeter galaxies $\left(\right.$ SMGs) ${ }^{2}$ (Jones et al., 2014, 2015).

The first subsample of WISE-selected galaxies were faint or undetectable flux densities in the $3.4 \mu \mathrm{m}$ (W1) and $4.6 \mu \mathrm{m}$ (W2) bands, and well detected fluxes in the W3 and/or W4 bands, with a radio blind selection, giving a "W1W2-dropout" selection yielding hot, dust obscured galaxies (Hot DOGs) (Eisenhardt et al., 2012; Wu et al., 2012).

The second subsample were found by Lonsdale et al. (2015), by combining WISE and National Radio Astronomy Observatory (NRAO) Very Large Array (VLA) Sky Survey (NVSS) (Condon et al., 1998) and/or Faint Images of the Radio Sky at Twentycm (FIRST) (Becker et al., 1995). They were selected in a similar method in the mid-IR, and are a similarly high luminosity, dustobscured population and in this paper are known as WISE/radio AGNs. The strong compact radio emission could be from AGN jets (Lonsdale et al., 2015).

\section{OVERDENSITY}

JCMT SCUBA-2 observations of all the WISE-selected AGN were in the "CV DAISY" mode that produces a uniformly deep coverage 3-arcmin diameter map (Holland et al., 2013). Seventeen companion sources were detected at $3 \sigma$ significance or above in 10 JCMT SCUBA-2 fields of Hot DOGs reported by Jones et al. (2014) with an average root mean square (RMS) noise of $1.8 \mathrm{mJy}$ beam $^{-1}$. Comparing these number counts to "blank field submm" surveys shows them to be overdense, with overdensity factor of 2-3, Jones et al. (2015).

Eighty-one companion sources were detected at $3 \sigma$ or greater significance in 30 WISE/radio-selected AGN fields reported by Jones et al. (2015) with average RMS noise of $2.1 \mathrm{mJy}^{\text {beam }}{ }^{-1}$. Comparing these number counts to "blank field submm" surveys shows them to be overdense, with overdensity factor of 5-6, Jones et al. (2014). The typical redshift of the 10 observed Hot DOGs is $z=2.7$ (Jones et al., 2014).

WISE/radio-selected AGN were found to have a higher density of SMGs when compared with Hot DOGs by a factor of $2.4 \pm 0.9$ (Jones et al., 2015). The WISE/radio AGNs have a lower redshift range, fewer of the WISE-selected AGNs are submm detected and lower total IR luminosities compared with Hot DOGs (Jones et al., 2014, 2015). The K-correction at wavelengths longer than $500 \mu \mathrm{m}$ remains approx. constant with increasing redshift. Due to this K-correction effect the SCUBA2 fraction of SMG detection should be independent of redshift.

\footnotetext{
${ }^{2}$ Submm galaxies (SMGs) were historically defined by having a submm flux density of $S_{850 \mu \mathrm{m}}>2 \mathrm{mJy}$. SMGs are massive gas-rich, high-redshift galaxies with high IR luminosities, $\mathrm{L}_{\mathrm{IR}} \geq 10^{12} \mathrm{~L}_{\odot}$, believed to be from starburst activity, with star formation rates (SFRs) of several $100-1,000 \mathrm{M}_{\odot} \mathrm{yr}^{-1}$ (Smail et al., 1997; Ivison et al., 1998; Eales et al., 1999; Smail et al., 2000; Blain et al., 2002; Pope et al., 2006; Casey et al., 2014; Swinbank et al., 2014). SMGs are enshrouded by dust and hence are faint in optical and near-IR wavelengths.
} 
The typical redshift of WISE/radio AGNs, $z=1.3$ (Jones et al., 2015).

\section{PROPERTIES OF COMPANION SOURCES}

The average submm flux density of SMGs around Hot DOGs is $S_{850 \mu \mathrm{m}}=6.2 \pm 1.8 \mathrm{mJy}$, which is comparable to SMGs around WISE/radio AGNs, $\mathrm{S}_{850 \mu \mathrm{m}}=7.2 \pm 2.1 \mathrm{mJy}$. Submm flux densities provide a reliable measurement of SFR (Alexander et al., 2016). The average $S F R$ is $\simeq 1,240 \mathrm{M}_{\odot} \mathrm{yr}^{-1}$ for $S M G$ around WISE/radio AGNs, slightly lower than the SFR $\simeq 1,460 \mathrm{M}_{\odot} \mathrm{yr}^{-1}$ for SMGs around Hot DOGs.

The star formation rate density (SFRD) represents the total star formation transpiring per unit time and volume at a given redshift. The SFRDs range for Hot DOGs from $1,523 \pm 30 \mathrm{M}_{\odot}$ $\mathrm{yr}^{-1} \mathrm{Mpc}^{-3}$ to $7,949 \pm 159 \mathrm{M}_{\odot} \mathrm{yr}^{-1} \mathrm{Mpc}^{-3}$, and average 3,533 $\mathrm{M}_{\odot} \mathrm{yr}^{-1} \mathrm{Mpc}^{-3}$. These are lower than WISE/radio AGNs with a range from $1,219 \pm 49 \mathrm{M}_{\odot} \mathrm{yr}^{-1} \mathrm{Mpc}^{-3}$ to $18,715 \pm 374 \mathrm{M}_{\odot}$ $\mathrm{yr}^{-1} \mathrm{Mpc}^{-3}$, and average 3,929 $\mathrm{M}_{\odot} \mathrm{yr}^{-1} \mathrm{Mpc}^{-3}$. These values are consistent to four Herschel Multitiered Extragalactic Survey (HerMES) clusters of dusty, star-forming galaxies at redshifts between $z=0.76$ to $z=2.26$, and other clusters with MIR/FIR measurements from the literature with SFRDs ranging from $\sim 200 \mathrm{M}_{\odot} \mathrm{yr}^{-1} \mathrm{Mpc}^{-3}$ to $\sim 3,000 \mathrm{M}_{\odot} \mathrm{yr}^{-1} \mathrm{Mpc}^{-3}$.

No counterparts to the companion sources from point sources were found in the third XMM-Newton companion Source Catalog, 3XMM-DR5 (Rosen et al., 2015). None of the companion sources around Hot DOGs or WISE/radio AGNs were detected at radio wavelengths in FIRST and/or NVSS, where the typical $1.4 \mathrm{GHz}$ detection limit was $1.0 \mathrm{mJy} / \mathrm{beam}$.

Both sets of companion sources have similar WISE colors, Jones et al. (2017). When comparing with the WISE color-color diagram of different galaxy populations in Figure 12 in Wright et al. (2010) and Figure 26 in Jarrett et al. (2011), the companion sources lie in both the starburst (star-forming) galaxy zone and AGN zone.

The Hot DOGs and WISE/radio AGNs are redder than the companion sources, due to the WISE-selected AGN having higher dust obscuration and/or a higher AGN contribution, and higher dust temperatures than that of their companion sources. Hot DOGs and WISE/radio AGNs are predominantly powered by AGN (Wu et al., 2012; Jones et al., 2014, 2015; Lonsdale et al., 2015; Tsai et al., 2015). SMGs are predominantly powered by star formation (Alexander et al., 2005), with cooler dust emission (20-50 K) (Hainline et al., 2009).

\section{CLUSTERING}

The angular two-point correlation function $\omega(\theta)$ is a statistical way to determine the clustering of galaxies in $2 \mathrm{D}$ space (Efstathiou et al., 1991; Connolly et al., 1998), using the angular version of the 3D spatial correlation function (Peebles, 1980). It is the excess probability of finding galaxies separated by $\theta$ above the probability with a random distribution. The popular estimators described by Landy and Szalay (1993) was used, see Figure 1.

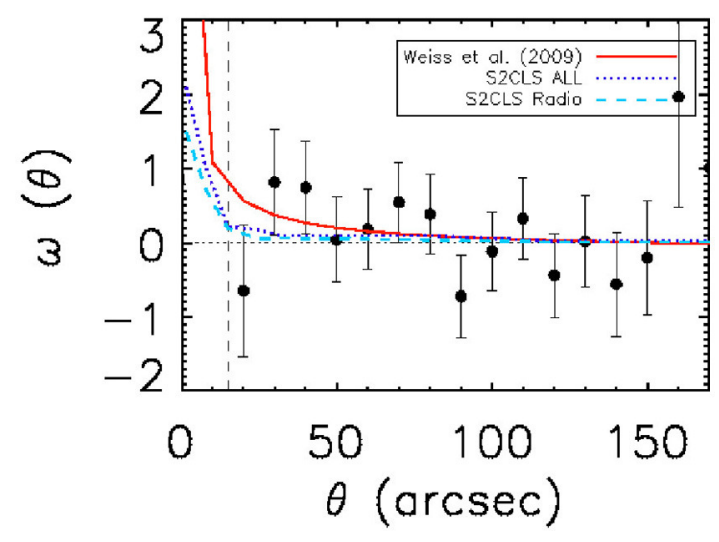

FIGURE 1 | Observed angular two-point correlation function using the Landy and Szalay (1993) equation, from Jones et al. (2017). The red solid curve shows the observed angular two-point correlation function for Weiß et al. (2009). The blue dotted line and cyan dashed line show the observed angular two-point correlation function for all the SMGs and the subset of radio-detected SMGs, respectively, in the S2CLS (Wilkinson et al., 2017). Black points represent the observed angular two-point correlation function for the companion sources detected around WISE/radio AGNs. The dashed line represents the JCMT SCUBA-2 $850 \mu \mathrm{m}$ beam size (15 arc s). There were not enough data for reliable and accurate results using the Hot DOGs.

The two-point angular clustering signal provided an upper limit to the strength of angular clustering (Jones et al., 2017), see Figure 1. Monte Carlo simulations showed no angular correlation, which could indicate protoclusters on scales larger than the SCUBA-2 1.5 arcmin scale maps.

Muldrew et al. (2015) investigated protoclusters and their environments using the Millennium Simulation. They found that protocluster structures are very extended at redshifts $z=2$, with $90 \%$ of their mass is dispersed across $\sim 30$ arcmin $\left(\sim 35 \mathrm{~h}^{-1} \mathrm{Mpc}\right.$ comoving). This suggests that many observations of protoclusters and high-redshift clusters are not imaging the full cluster. This could explain why there is an upper limit of angular clustering in the Hot DOGs and WISE/radio AGNs fields on $\sim 1.5 \mathrm{arcmin}$ scales. Alternatively, the cluster might be peaked substantially off-center from the WISE target. Further and wider observations of companion sources in the fields around WISE/radio AGN are needed to determine the clustering of WISEselected AGN.

\section{DISCUSSION/CONCLUSION}

1. Hot DOGs and WISE/radio AGNs have very high total IR luminosities, hot dust temperatures (60-120 K), and SEDs that are not well fitted by many standard AGN templates due to excess mid-IR emission and less submm emission (Jones et al., 2014, 2015).

2. Hot DOGs and WISE/radio AGNs appear to be consistent with the same population of very luminous, AGNdominated galaxies but are different redshifts. They could be a new transient phase of the major merger model (Jones et al., 2014, 2015). 
3. WISE/radio AGNs are typically at a lower redshift $(z=$ 1.7) than Hot DOGs $(z=2.7)$. The lower redshift WISE/radio AGNs appear to reside in higher density regions compared with higher redshift Hot DOGs. This could be due to differences in redshift and/or radio emission. However, more observations are needed because only 10 targets in each sample have known redshifts, Jones et al. (2017).

4. The space densities of SMGs around the WISEselected AGNs were found to overdense compared to normal star-forming galaxies and SMGs in the S2CLS, Jones et al. (2017).

5. There is an upper limit to the strength of angular clustering of the companion SMG sources in Hot DOGs and WISE/radio AGNs on SCUBA-2 1.5 arcmin scales. The typical separations when compared to Monte Carlo simulations showed no angular clustering. This is an agreement with the cumulative fraction of companion sources in different radii from the WISE target. This could be because they are satellite galaxies in the massive halo or that the protocluster is on bigger scales (up to $\sim 30$ arcmin) and we are not fully probing the protocluster, Jones et al. (2017).

6. The SMGs around WISE/radio AGNs 18\% higher SFRs than SMGs around Hot DOGs, Jones et al. (2017).

7. The SFRDs of the WISE-selected AGNs are higher than field galaxies, and consistent with values for known clusters of dusty galaxies, Jones et al. (2017).

8. The companion sources detected around Hot DOGs and WISE/radio AGNs have WISE colors consistent with starforming galaxies and mid-IR to submm ratios not consistent with AGN dominated sources. This could imply that they are all consistent with SMGs, Jones et al. (2017).

9. All the companion sources have bluer mid-IR positions in the WISE color-color plot compared with Hot DOGs and

\section{REFERENCES}

Alexander, D. M., Simpson, J. M., Harrison, C. M., Mullaney, J. R., Smail, I., Geach, J. E., et al. (2016). ALMA observations of a $\mathrm{z} \approx 3.1$ protocluster: star formation from active galactic nuclei and Lyman-alpha blobs in an overdense environment. Month. Notices R. Astron. Soc. 461, 2944-2952. doi: 10.1093/mnras/stw1509

Alexander, D. M., Smail, I., Bauer, F. E., Chapman, S. C., Blain, A. W., Brandt, W. N., et al. (2005). Rapid growth of black holes in massive star-forming galaxies. Nature 434, 738-740. doi: 10.1038/nature03473

Avila, S., Knebe, A., Pearce, F. R., Schneider, A., Srisawat, C., Thomas, P. A., et al. (2014). SUSSING MERGER TREES: the influence of the halo finder. Month. Notices R. Astron. Soc. 441, 3488-3501. doi: 10.1093/mnras/stu799

Becker, R. H., White, R. L., and Helfand, D. J. (1995). The FIRST survey: Faint images of the radio sky at twenty centimeters. Astrophys. J. 450:559. doi: $10.1086 / 176166$

Best, P. N., Kauffmann, G., Heckman, T. M., Brinchmann, J., Charlot, S., Ivezić, Ž., et al. (2005). The host galaxies of radio-loud active galactic nuclei: mass dependences, gas cooling and active galactic nuclei feedback. Month. Notices R. Astron. Soc. 362, 25-40. doi: 10.1111/j.1365-2966.2005. 09192.x

Bett, P., Eke, V., Frenk, C. S., Jenkins, A., Helly, J., and Navarro, J. (2007). The spin and shape of dark matter haloes in the Millennium simulation of a $\Lambda$ cold dark matter universe. Month. Notices R. Astron. Soc. 376, 215-232. doi: 10.1111/j.1365-2966.2007.11432.x
WISE/radio AGNs, which implies cooler dust temperatures than 60-120 K, Jones et al. (2017).

10. Hot DOGs and WISE/radio AGNs appear to be good indicators of overdense environments of active galaxies in arcmin scales, Jones et al. (2017).

11. Further spectroscopic redshift data of the WISE-selected targets and their companion SMG sources are needed.

12. Further submm data of WISE-selected targets are needed to increase the sample size of WISE-selected targets. Also high-resolution ALMA data are needed to resolve the galaxies to see if there are multiple components for example of WISEselected Hot DOG W2026+0716.

\section{AUTHOR CONTRIBUTIONS}

The author confirms being the sole contributor of this work and approved it for publication.

\section{ACKNOWLEDGMENTS}

This publication makes use of data products from the Widefield Infrared Survey Explorer, which is a joint project of the University of California, Los Angeles, and the Jet Propulsion Laboratory/California Institute of Technology, funded by the National Aeronautics and Space Administration. The James Clerk Maxwell Telescope has historically been operated by the Joint Astronomy Centre on behalf of the Science and Technology Facilities Council of the United Kingdom, the National Research Council of Canada and the Netherlands Organization for Scientific Research. Additional funds for the construction of SCUBA-2 were provided by the Canada Foundation for Innovation. The program IDs under which the data were obtained were M12AU10, M12BU07, and M13BU02.

Blain, A. W., Chapman, S. C., Smail, I., and Ivison, R. (2004). Clustering of Submillimeter-selected Galaxies. Astrophys. J. 611, 725-731. doi: $10.1086 / 422353$

Blain, A. W., Smail, I., Ivison, R. J., Kneib, J.-P., and Frayer, D. T. (2002). Submillimeter galaxies. Phys. Rep. 369, 111-176. doi: 10.1016/S0370-1573(02)00134-5

Borys, C., Scott, D., Chapman, S., Halpern, M., Nandra, K., and Pope, A. (2004). The hubble deep field north SCUBA super-map - II. Multiwavelength properties. Month. Notices R. Astron. Soc. 355, 485-503. doi: 10.1111/j.1365-2966.2004.08335.x

Bridge, C. R., Blain, A., Borys, C. J. K., Petty, S., Benford, D., Eisenhardt, P., et al. (2013). A new population of high-z, dusty Ly $\alpha$ emitters and blobs discovered by WISE: feedback caught in the act? Astrophys. J. 769:91. doi: 10.1088/0004-637X/769/2/91

Casey, C. M., Scoville, N. Z., Sanders, D. B., Lee, N., Cooray, A., Finkelstein, S. L., et al. (2014). Are dusty galaxies blue? Insights on UV attenuation from dust-selected galaxies. Astrophys. J. 796:95. doi: 10.1088/0004-637X/796/2/95

Chapman, S. C., Blain, A., Ibata, R., Ivison, R. J., Smail, I., and Morrison, G. (2009). Do submillimeter galaxies really trace the most massive dark-matter halos? Discovery of a high-z cluster in a highly active phase of evolution. Astrophys. J. 691, 560-568. doi: 10.1088/0004-637X/691/1/560

Coil, A. L. (2013). The Large-Scale Structure of the Universe. Dordrecht: Springer Science+Business Media.

Coil, A. L., Georgakakis, A., Newman, J. A., Cooper, M. C., Croton, D., Davis, M., et al. (2009). AEGIS: the clustering of X-ray active galactic 
nucleus relative to galaxies at $\mathrm{z} \sim 1$. Astrophys. J. 701, 1484-1499. doi: 10.1088/0004-637X/701/2/1484

Coldwell, G. V., and Lambas, D. G. (2006). Properties of galaxies in Sloan Digital Sky Survey quasar environments at $\mathrm{z}<0.2$. Month. Notices R. Astron. Soc. 371, 786-792. doi: 10.1111/j.1365-2966.2006.10712.x

Condon, J. J., Cotton, W. D., Greisen, E. W., Yin, Q. F., Perley, R. A., Taylor, G. B., et al. (1998). The NRAO VLA sky survey. Astron. J. 115, 1693-1716. doi: $10.1086 / 300337$

Connolly, A. J., Szalay, A. S., and Brunner, R. J. (1998). Evolution of the angular correlation function. Astrophys. J. Lett. 499, L125-L129. doi: 10.1086/311362

Cooray, A., Amblard, A., Wang, L., Arumugam, V., Auld, R., Aussel, H., et al. (2010). HerMES: Halo occupation number and bias properties of dusty galaxies from angular clustering measurements. Astron. Astrophys. 518:L22. doi: 10.1051/0004-6361/201014597

Dannerbauer, H., Kurk, J. D., De Breuck, C., Wylezalek, D., Santos, J. S., Koyama, Y., et al. (2014). An excess of dusty starbursts related to the Spiderweb galaxy. Astron. Astrophys. 570:A55. doi: 10.1051/0004-6361/201423771

Davis, M., Efstathiou, G., Frenk, C. S., and White, S. D. M. (1985). The evolution of large-scale structure in a universe dominated by cold dark matter. Astrophys. J. 292, 371-394. doi: 10.1086/163168

De Breuck, C., Bertoldi, F., Carilli, C., Omont, A., Venemans, B., Röttgering, H., et al. (2004). A multi-wavelength study of the proto-cluster surrounding the $\mathrm{z}=4.1$ radio galaxy TN J1338-1942. Astron. Astrophys. 424, 1-12. doi: 10.1051/0004-6361:20035885

Degraf, C., Di Matteo, T., and Springel, V. (2011). Black hole clustering in cosmological hydrodynamic simulations: evidence for mergers. Month. Notices R. Astron. Soc. 413, 1383-1394. doi: 10.1111/j.1365-2966.2011.18221.x

Donoso, E., Li, C., Kauffmann, G., Best, P. N., and Heckman, T. M. (2010). Clustering of radio galaxies and quasars. Month. Notices R. Astron. Soc. 407, 1078-1089. doi: 10.1111/j.1365-2966.2010.16907.x

Donoso, E., Yan, L., Stern, D., and Assef, R. J. (2014). The angular clustering of WISE-selected active galactic nuclei: different halos for obscured and unobscured active galactic nuclei. Astrophys. J. 789:44. doi: 10.1088/0004-637X/789/1/44

Eales, S., Lilly, S., Gear, W., Dunne, L., Bond, J. R., Hammer, F., et al. (1999). The Canada-UK deep submillimeter survey: first submillimeter images, the source counts, and resolution of the background. Astrophys. J. 515, 518-524. doi: $10.1086 / 307069$

Efstathiou, G., Bernstein, G., Tyson, J. A., Katz, N., and Guhathakurta, P. (1991). The clustering of faint galaxies. Astrophys. J. Lett. 380, L47-L50. doi: $10.1086 / 186170$

Eisenhardt, P. R. M., Wu, J., Tsai, C.-W., Assef, R., Benford, D., Blain, A., et al. (2012). The first hyper-luminous infrared galaxy discovered by WISE. Astrophys. J. 755:173. doi: 10.1088/0004-637X/755/2/173

Fakhouri, O., and Ma, C.-P. (2009). Environmental dependence of dark matter halo growth - I. Halo merger rates. Month. Notices R. Astron. Soc. 394, 18251840. doi: 10.1111/j.1365-2966.2009.14480.x

Fakhouri, O., Ma, C.-P., and Boylan-Kolchin, M. (2010). The merger rates and mass assembly histories of dark matter haloes in the two Millennium simulations. Month. Notices R. Astron. Soc. 406, 2267-2278. doi: $10.1111 / j .1365-2966.2010 .16859 . x$

Falder, J. T., Stevens, J. A., Jarvis, M. J., Hardcastle, M. J., Lacy, M., McLure, R. J., et al. (2010). The environments of $\mathrm{z} \sim 1$ active galactic nuclei at $3.6 \mu \mathrm{m}$. Month. Notices R. Astron. Soc. 405, 347-358. doi: 10.1111/j.1365-2966.2010. 16444.x

Faltenbacher, A., and White, S. D. M. (2010). Assembly bias and the dynamical structure of dark matter halos. Astrophys. J. 708, 469-473. doi: 10.1088/0004-637X/708/1/469

Fanidakis, N., Baugh, C. M., Benson, A. J., Bower, R. G., Cole, S., Done, C., et al. (2011). Grand unification of AGN activity in the $\Lambda$ CDM cosmology. Month. Notices R. Astron. Soc. 410, 53-74. doi: 10.1111/j.1365-2966.2010. 17427.x

Farrah, D., Lonsdale, C. J., Borys, C., Fang, F., Waddington, I., Oliver, S., et al. (2006). The spatial clustering of ultraluminous infrared galaxies over $1.5<\mathrm{z}<$ 3. Astrophys. J. Lett. 641, L17-L20. doi: 10.1086/503769

Galametz, A., Stern, D., De Breuck, C., Hatch, N., Mayo, J., Miley, G., et al. (2012). The mid-infrared environments of high-redshift radio galaxies. Astrophys. J. 749:169. doi: 10.1088/0004-637X/749/2/169
Galametz, A., Vernet, J., De Breuck, C., Hatch, N. A., Miley, G. K., Kodama, T., et al. (2010). Galaxy protocluster candidates at $1.6<\mathrm{z}<2$. Astron. Astrophys. 522:A58. doi: 10.1051/0004-6361/201015035

Gao, L., Yoshida, N., Abel, T., Frenk, C. S., Jenkins, A., and Springel, V. (2007). The first generation of stars in the $\Lambda$ cold dark matter cosmology. Month. Notices $R$. Astron. Soc. 378, 449-468. doi: 10.1111/j.1365-2966.2007.11814.x

Georgakakis, A., Nandra, K., Laird, E. S., Cooper, M. C., Gerke, B. F., Newman, J. A., et al. (2007). AEGIS: the environment of X-ray sources at $z^{\sim} 1$. Astrophys. J. Lett. 660, L15-L18. doi: 10.1086/517920

Gilli, R., Comastri, A., and Hasinger, G. (2007). The synthesis of the cosmic Xray background in the Chandra and XMM-Newton era. Astron. Astrophys. 463, 79-96. doi: 10.1051/0004-6361:20066334

Hainline, L. J., Blain, A. W., Smail, I., Frayer, D. T., Chapman, S. C., Ivison, R. J., et al. (2009). A mid-infrared imaging survey of submillimeter-selected galaxies with the spitzer space telescope. Astrophys. J. 699, 1610-1632. doi: 10.1088/0004-637X/699/2/1610

Hatch, N. A., De Breuck, C., Galametz, A., Miley, G. K., Overzier, R. A., Röttgering, H. J. A., et al. (2011). Galaxy protocluster candidates around z 2.4 radio galaxies. Month. Notices R. Astron. Soc. 410, 1537-1549. doi: 10.1111/j.1365-2966.2010.17538.x

Hatch, N. A., Wylezalek, D., Kurk, J. D., Stern, D., De Breuck, C., Jarvis, M. J., et al. (2014). Why $\mathrm{z}>1$ radio-loud galaxies are commonly located in protoclusters. Month. Notices R. Astron. Soc. 445, 280-289. doi: 10.1093/mnras/stu1725

Hennawi, J. F., Prochaska, J. X., Burles, S., Strauss, M. A., Richards, G. T., Schlegel, D. J., et al. (2006). Quasars probing quasars. I. Optically thick absorbers near luminous quasars. Astrophys. J. 651, 61-83. doi: 10.1086/507069

Hickox, R. C., Jones, C., Forman, W. R., Murray, S. S., Kochanek, C. S., Eisenstein, D., et al. (2009). Host galaxies, clustering, eddington ratios, and evolution of radio, X-ray, and infrared-selected AGNs. Astrophys. J. 696, 891-919. doi: 10.1088/0004-637X/696/1/891

Hickox, R. C., Wardlow, J. L., Smail, I., Myers, A. D., Alexander, D. M., Swinbank, A. M., et al. (2012). The LABOCA survey of the Extended Chandra Deep FieldSouth: clustering of submillimetre galaxies. Month. Notices R. Astron. Soc. 421, 284-295. doi: 10.1111/j.1365-2966.2011.20303.x

Holland, W. S., Bintley, D., Chapin, E. L., Chrysostomou, A., Davis, G. R., Dempsey, J. T., et al. (2013). SCUBA-2: the 10000 pixel bolometer camera on the James Clerk Maxwell Telescope. Month. Notices R. Astron. Soc. 430, 2513-2533. doi: 10.1093/mnras/sts612

Ivison, R. J., Smail, I., Le Borgne, J.-F., Blain, A. W., Kneib, J.-P., Bezecourt, J., et al. (1998). A hyperluminous galaxy at $\mathrm{z}=2.8$ found in a deep submillimetre survey. Month. Notices R. Astron. Soc. 298, 583-593. doi: 10.1046/j.1365-8711.1998.01677.x

Jarrett, T. H., Cohen, M., Masci, F., Wright, E., Stern, D., Benford, D., et al. (2011). The Spitzer-WISE survey of the ecliptic poles. Astrophys. J. 735:112. doi: $10.1088 / 0004-637 X / 735 / 2 / 112$

Jing, Y. P., Suto, Y., and Mo, H. J. (2007). The dependence of dark halo clustering on formation epoch and concentration parameter. Astrophys. J. 657, 664-668. doi: $10.1086 / 511130$

Jones, S. F., Blain, A. W., Assef, R. J., Eisenhardt, P., Lonsdale, C., Condon, J., et al. (2017). Overdensities of SMGs around WISE-selected, ultraluminous, high-redshift AGNs. Month. Notices R. Astron. Soc. 469, 4565-4577. doi: $10.1093 / \mathrm{mnras} / \mathrm{stx} 1141$

Jones, S. F., Blain, A. W., Lonsdale, C., Condon, J., Farrah, D., Stern, D., et al. (2015). Submillimetre observations of WISE/radio-selected AGN and their environments. Month. Notices R. Astron. Soc. 448, 3325-3338. doi: $10.1093 / \mathrm{mnras} / \mathrm{stv} 214$

Jones, S. F., Blain, A. W., Stern, D., Assef, R. J., Bridge, C. R., Eisenhardt, P., et al. (2014). Submillimetre observations of WISE-selected high-redshift, luminous, dusty galaxies. Month. Notices R. Astron. Soc. 443, 146-157. doi: $10.1093 / \mathrm{mnras} / \mathrm{stu} 1157$

Kauffmann, G., White, S. D. M., and Guiderdoni, B. (1993). The formation and evolution of galaxies within merging dark matter haloes. Month. Notices $R$. Astron. Soc. 264:201. doi: 10.1093/mnras/264.1.201

Kauffmann, G., White, S. D. M., Heckman, T. M., Ménard, B., Brinchmann, J., Charlot, S., et al. (2004). The environmental dependence of the relations between stellar mass, structure, star formation and nuclear activity in galaxies. Month. Notices R. Astron. Soc. 353, 713-731. doi: 10.1111/j.1365-2966.2004.08117.x 
Kellermann, K. I., Sramek, R., Schmidt, M., Shaffer, D. B., and Green, R. (1989). VLA observations of objects in the Palomar Bright Quasar Survey. Astron. J. 98, 1195-1207. doi: 10.1086/115207

Landy, S. D., and Szalay, A. S. (1993). Bias and variance of angular correlation functions. Astrophys. J. 412, 64-71. doi: 10.1086/172900

Lonsdale, C. J., Lacy, M., Kimball, A. E., Blain, A., Whittle, M., Wilkes, B., et al. (2015). Radio jet feedback and star formation in heavily obscured, hyperluminous quasars at redshifts $\sim 0.5-3$. I. ALMA observations. Astrophys. J. 813:45. doi: 10.1088/0004-637X/813/1/45

Magliocchetti, M., and Brüggen, M. (2007). The interplay between radio galaxies and cluster environment. Month. Notices R. Astron. Soc. 379, 260-274. doi: 10.1111/j.1365-2966.2007.11939.x

Matthews, T. A., Morgan, W. W., and Schmidt, M. (1964). A discussion of galaxies indentified with radio sources. Astrophys. J. 140:35. doi: 10.1086/147890

Mayo, J. H., Vernet, J., De Breuck, C., Galametz, A., Seymour, N., and Stern, D. (2012). Overdensities of $24 \mu \mathrm{m}$ sources in the vicinities of highredshift radio galaxies. Astron. Astrophys. 539:A33. doi: 10.1051/0004-6361/201 118254

Miller, C. J., Nichol, R. C., Gómez, P. L., Hopkins, A. M., and Bernardi, M. (2003). The environment of active galactic nuclei in the sloan digital sky survey. Astrophys. J. 597, 142-156. doi: 10.1086/378383

Miller, J. S., and Goodrich, R. W. (1990). Spectropolarimetry of high-polarization Seyfert 2 galaxies and unified Seyfert theories. Astrophys. J. 355, 456-467. doi: $10.1086 / 168780$

Mo, H. J., and White, S. D. M. (2002). The abundance and clustering of dark haloes in the standard $\Lambda$ CDM cosmogony. Month. Notices R. Astron. Soc. 336, 112-118. doi: 10.1046/j.1365-8711.2002.05723.x

Muldrew, S. I., Hatch, N. A., and Cooke, E. A. (2015). What are protoclusters? - Defining high-redshift galaxy clusters and protoclusters. Month. Notices $R$. Astron. Soc. 452, 2528-2539. doi: 10.1093/mnras/stv1449

Myers, A. D., Brunner, R. J., Nichol, R. C., Richards, G. T., Schneider, D. P., and Bahcall, N. A. (2007). Clustering analyses of 300,000 photometrically classified quasars. I. Luminosity and redshift evolution in quasar bias. Astrophys. J. 658, 85-98. doi: 10.1086/511519

Navarro, J. F., Frenk, C. S., and White, S. D. M. (1996). The structure of cold dark matter halos. Astrophys. J. 462:563. doi: 10.1086/177173

Peebles, P. J. E. (1980). The Large-Scale Structure of the Universe. Princeton, NJ: Princeton University Press.

Pope, A., Scott, D., Dickinson, M., Chary, R.-R., Morrison, G., Borys, C., et al. (2006). The hubble deep field-north SCUBA super-map - IV. Characterizing submillimetre galaxies using deep Spitzer imaging. Month. Notices R. Astron. Soc. 370, 1185-1207. doi: 10.1111/j.1365-2966.2006.10575.x

Rigby, E. E., Hatch, N. A., Röttgering, H. J. A., Sibthorpe, B., Chiang, Y. K., Overzier, R., et al. (2014). Searching for large-scale structures around highredshift radio galaxies with Herschel. Month. Notices R. Astron. Soc. 437, 1882-1893. doi: $10.1093 / \mathrm{mnras} / \mathrm{stt} 2019$

Rosen, S., Watson, M., Pye, J., Webb, N., Schwope, A., Freyberg, M., et al. (2015). "The 3XMM-DR4 catalogue," in Astronomical Data Analysis Software an Systems XXIV (ADASS XXIV), Vol. 495 of Astronomical Society of the Pacific Conference Series, eds A. R. Taylor and E. Rosolowsky (San Francisco, CA), 319.

Ruderman, J. T., and Ebeling, H. (2005). The origin of the spatial distribution of $\mathrm{X}$-ray-luminous active galactic nuclei in massive galaxy clusters. Astrophys. J. Lett. 623, L81-L84. doi: 10.1086/430131

Scott, S. E., Dunlop, J. S., and Serjeant, S. (2006). A combined reanalysis of existing blank-field SCUBA surveys: comparative $850-\mu \mathrm{m}$ source lists, combined number counts, and evidence for strong clustering of the bright submillimetre galaxy population on arcminute scales. Month. Notices R. Astron. Soc. 370, 1057-1105. doi: 10.1111/j.1365-2966.2006. 10478.x

Serber, W., Bahcall, N., Ménard, B., and Richards, G. (2006). The small-scale environment of quasars. Astrophys. J. 643, 68-74. doi: 10.1086/501443

Smail, I., Ivison, R. J., and Blain, A. W. (1997). A deep sub-millimeter survey of lensing clusters: a new window on galaxy formation and evolution. Astrophys. J. Lett. 490:L5. doi: 10.1086/311017

Smail, I., Ivison, R. J., Owen, F. N., Blain, A. W., and Kneib, J.-P. (2000). Radio constraints on the identifications and redshifts of submillimeter galaxies. Astrophys. J. 528, 612-616. doi: 10.1086/308226
Springel, V., White, S. D. M., Jenkins, A., Frenk, C. S., Yoshida, N., Gao, L., et al. (2005). Simulations of the formation, evolution and clustering of galaxies and quasars. Nature 435, 629-636. doi: 10.1038/nature03597

Stevens, J. A., Ivison, R. J., Dunlop, J. S., Smail, I. R., Percival, W. J., Hughes, D. H., et al. (2003). The formation of cluster elliptical galaxies as revealed by extensive star formation. Nature 425, 264-267. doi: 10.1038/nature01976

Stevens, J. A., Jarvis, M. J., Coppin, K. E. K., Page, M. J., Greve, T. R., Carrera, F. J., et al. (2010). An excess of star-forming galaxies in the fields of high-redshift QSOs. Month. Notices R. Astron. Soc. 405, 2623-2638. doi: 10.1111/j.1365-2966.2010.16641.x

Strauss, M. A., and Willick, J. A. (1995). The density and peculiar velocity fields of nearby galaxies. Phys. Rep. 261, 271-431. doi: 10.1016/0370-1573(95)00013-7

Swinbank, A. M., Simpson, J. M., Smail, I., Harrison, C. M., Hodge, J. A., Karim, A., et al. (2014). An ALMA survey of sub-millimetre Galaxies in the Extended Chandra Deep Field South: the far-infrared properties of SMGs. Month. Notices R. Astron. Soc. 438, 1267-1287. doi: 10.1093/mnras/ stt2273

Tsai, C.-W., Eisenhardt, P. R. M., Wu, J., Stern, D., Assef, R. J., Blain, A. W., et al. (2015). The most luminous galaxies discovered by WISE. Astrophys. J. 805:90. doi: $10.1088 / 0004-637 \mathrm{X} / 805 / 2 / 90$

Umehata, H., Tamura, Y., Kohno, K., Hatsukade, B., Scott, K. S., Kubo, M., et al. (2014). AzTEC/ASTE 1.1-mm survey of SSA22: counterpart identification and photometric redshift survey of submillimetre galaxies. Month. Notices $R$. Astron. Soc. 440, 3462-3478. doi: 10.1093/mnras/stu447

Urry, C. M., and Padovani, P. (1995). Unified Schemes for Radio-Loud Active Galactic Nuclei. Publ. Astron. Soc. Pacific 107:803. doi: 10.1086/133630

Wake, D. A., Franx, M., and van Dokkum, P. G. (2012). Which galaxy property is the best indicator of its host dark matter halo properties? arXiv:1201.1913.

Wechsler, R. H., Zentner, A. R., Bullock, J. S., Kravtsov, A. V., and Allgood, B. (2006). The dependence of halo clustering on halo formation history, concentration, and occupation. Astrophys. J. 652, 71-84. doi: 10.1086/507120

Weiß, A., Kovács, A., Coppin, K., Greve, T. R., Walter, F., Smail, I., et al. (2009). The large apex bolometer camera survey of the extended chandra deep field south. Astrophys. J. 707, 1201-1216. doi: 10.1088/0004-637X/707/2/1201

Wetzel, A. R., Cohn, J. D., White, M., Holz, D. E., and Warren, M. S. (2007). The clustering of massive halos. Astrophys. J. 656, 139-147. doi: 10.1086/510444

Wilkinson, A., Almaini, O., Chen, C.-C., Smail, I., Arumugam, V., Blain, A., et al. (2017). The SCUBA-2 cosmology legacy survey: the clustering of submillimetre galaxies in the UKIDSS UDS field. Month. Notices R. Astron. Soc. 464, $1380-$ 1392. doi: $10.1093 / \mathrm{mnras} / \mathrm{stw} 2405$

Wright, E. L., Eisenhardt, P. R. M., Mainzer, A. K., Ressler, M. E., Cutri, R. M., Jarrett, T., et al. (2010). The wide-field infrared survey explorer (WISE): mission description and initial on-orbit performance. Astron. J. 140, 18681881. doi: $10.1088 / 0004-6256 / 140 / 6 / 1868$

Wu, J., Tsai, C.-W., Sayers, J., Benford, D., Bridge, C., Blain, A., et al. (2012). Submillimeter follow-up of WISE-selected hyperluminous galaxies. Astrophys. J. 756:96. doi: 10.1088/0004-637X/756/1/96

Wylezalek, D., Galametz, A., Stern, D., Vernet, J., De Breuck, C., Seymour, N., et al. (2013). Galaxy clusters around radio-loud active galactic nuclei at 1.3 $<\mathrm{z}<3.2$ as seen by spitzer. Astrophys. J. 769:79. doi: 10.1088/0004-637X/ $769 / 1 / 79$

Wylezalek, D., Vernet, J., De Breuck, C., Stern, D., Brodwin, M., Galametz, A., et al. (2014). The galaxy cluster mid-infrared luminosity function at $1.3<\mathrm{z}<3.2$. Astrophys. J. 786:17. doi: 10.1088/0004-637X/786/1/17

Conflict of Interest Statement: The author declares that the research was conducted in the absence of any commercial or financial relationships that could be construed as a potential conflict of interest.

The reviewer DB and handling Editor declared their shared affiliation.

Copyright $\odot 2017$ Jones. This is an open-access article distributed under the terms of the Creative Commons Attribution License (CC BY). The use, distribution or reproduction in other forums is permitted, provided the original author(s) or licensor are credited and that the original publication in this journal is cited, in accordance with accepted academic practice. No use, distribution or reproduction is permitted which does not comply with these terms. 